\title{
Intervenções para enfrentamento do abuso de álcool: revisão integrativa*
}

\section{Interventions to cope with alcohol abuse: integrative review}

Fernanda Jorge Guimarães ${ }^{1}$, Ana Fátima Carvalho Fernandes ${ }^{2}$, Lorita Marlena Freitag Pagliuca ${ }^{3}$

\footnotetext{
* Recorte da Tese de Doutorado, defendida em 2014, intitulada "Validação de tecnologia assistiva sobre substâncias psicoativas para pessoas com deficiência visual".

${ }^{1}$ Enfermeira, Doutora em Enfermagem. Professora Assistente da Universidade Federal de Pernambuco. Recife, PE, Brasil. E-mail: ferjorgui@hotmail.com.

${ }^{2}$ Enfermeira, Doutora em Enfermagem. Professor Titular da Universidade Federal do Ceará (UFC). Fortaleza, CE, Brasil. E-mail: afcana@ufc.br.

${ }^{3}$ Enfermeira, Doutora em Enfermagem. Professor Titular da UFC. Fortaleza, CE, Brasil. E-mail: pagliuca@ufc.br.
}

\section{RESUMO}

O álcool é a droga mais consumida no mundo, que pode gerar problemas sociais e de saúde, afetando usuários, pessoas que com estes convivem e a sociedade em geral. O objetivo foi identificar melhores evidências em intervenções para redução do abuso de álcool. Revisão integrativa de literatura, realizada na LILACS, CINAHL, PUBMED e SCOPUS, por meio dos descritores intervention studies e alcoholism. Os 19 artigos selecionados, em sua maioria, foram classificados como nível dois de evidência, envolviam intervenções com usuários de álcool, destacando-se como as mais eficientes as intervenções breves, intervenções baseadas na internet e aconselhamento. Embora não se comprovou a cessação do uso do álcool a partir das intervenções, os resultados apontam redução significativa do consumo, aumento na disponibilidade para mudança do ato de beber e impacto efetivo das intervenções breves quando comparadas aos tratamentos usuais. As intervenções breves constituem as melhores intervenções para redução do abuso de álcool.

Descritores: Alcoolismo; Estudos de Intervenção; Enfermagem; Promoção da Saúde.

\section{ABSTRACT}

Alcohol is the most consumed drug in the world, which could generate social and health problems, affecting users, people living with them and the society in general. The aim was to identify the best evidence of interventions to reduce alcohol abuse. An integrative review of the literature, conducted on LILACS, CINAHL, PUBMED and SCOPUS, through the descriptors "intervention studies" and "alcoholism". Nineteen articles were selected, most of them classified as two regarding level of evidence. They involved interventions with alcohol users, the most efficient were short interventions, internet-based interventions and counselling. Although cessation of alcohol use was not proved through the interventions, results point to a significant reduction in consumption, increase of the availability to change drinking habit and effective impact of short interventions when compared to usual treatments. Short interventions constitute the best interventions to reduce alcohol abuse.

Descriptors: Alcoholism; Intervention Studies; Nursing; Health Promotion. 


\section{INTRODUÇÃO}

O álcool vem se destacando como a droga mais consumida no mundo, como também no país, e está associado a danos à saúde da população, acidentes, problemas sociais, dentre outros. Indivíduos com idade acima de 15 anos bebem, em média, 6,2 litros de álcool puro por ano, ou seja, 13,5 gramas de álcool puro por $\operatorname{dia}^{(1)}$.

No Brasil, o alcoolismo avança, significativamente, com $63 \%$ da população brasileira consumidora de algum tipo de bebida alcoólica, $12 \%$ dependentes e mais de $10 \%$ relacionam-se à ocorrência de morbidade e mortalidade $^{(2)}$. O número de adultos que bebe pelo menos uma vez por semana é de $54 \%^{(3)}$. O abuso do álcool pode, também, estar conexo ao consumo de drogas ilícitas.

Para enfrentar a problemática exposta, é importante a implementação e avaliação de intervenções para a redução do abuso do álcool. Essas intervenções constituem evidências relevantes para a prática de profissionais de saúde.

Diante do exposto, emergiu o interesse no desenvolvimento deste estudo, pois o levantamento de evidências oriundas de estudos com rigor metodológico se justifica pela relevância que essas evidências trazem para a prática, especialmente no atual cenário brasileiro, em que há maior atenção para a problemática do álcool, crack e outras drogas.

Dessa forma, optou-se pela revisão integrativa sobre as intervenções para redução do consumo de álcool, por entender que a identificação das melhores evidências contribuem para o enfrentamento desta problemática e como suporte às ações práticas de promoção da saúde.

Ademais, uma consulta na Cochrane database mostrou que os estudos relacionados a temática investigam intervenções específicas para determinados grupos como jovens, idosos e gestantes, não havendo, portanto, estudos na perspectiva aqui proposta.
Assim, o objetivo do estudo consistiu em identificar melhores evidências em intervenções para redução do abuso de álcool.

\section{MÉTODO}

Para a realização deste estudo, seguiram-se as etapas da revisão integrativa: estabelecimento de hipótese e objetivos da revisão; definição de critérios de inclusão e exclusão de artigos (seleção da amostra); definição das informações a serem extraídas dos artigos selecionados; análise dos resultados; discussão e apresentação dos resultados $^{(4)}$.

Para nortear esta revisão integrativa, formulou-se a seguinte questão: quais intervenções, a partir das evidências científicas, são mais eficientes para redução do abuso de álcool?

Os critérios de inclusão dos artigos foram: ser artigo completo de pesquisa, estar publicado nos idiomas inglês, português ou espanhol, no período de 2005 a 2015; apresentar intervenções para redução do abuso de álcool e possuir nível de evidência um ou dois. Não foram definidos critérios de exclusão.

As evidências científicas são classificadas de acordo com a fonte dos dados e a solidez das informações fornecidas. Deste modo, para este estudo, optou-se pelo levantamento das evidências originadas de revisões sistemáticas ou meta-análise de ensaios clínicos randomizados controlados, nível 1 , e de ensaios clínicos randomizados, nível 2 de evidência ${ }^{(5)}$, por entender que tais fontes fornecem melhores evidências para a prática. Os artigos foram pesquisados nas bases de dados LILACS, CINAHL, PUBMED e SCOPUS, utilizando os descritores: estudos de intervenção e alcoolismo; Intervention Studies e Alcoholism.

Para a seleção dos artigos, inicialmente, realizou-se a busca nas bases com os descritores citados anteriormente. A segunda etapa consistiu em identificar o idioma dos artigos encontrados. Em seguida, realizouse a leitura dos resumos e foram excluídos os artigos que não tinham relação com o tema, não apresentaram 
intervenção e não possuíam nível de evidência um ou dois. Um artigo se encontrava presente em duas das bases pesquisadas. A Tabela 1 apresenta a distribuição dos artigos de acordo com os critérios de busca e o descarte nas bases de dados pesquisadas.

Tabela 1: Distribuição dos artigos, de acordo com as bases de dados LILACS, CINAHL, PUBMED e SCOPUS. Fortaleza, CE, Brasil, 2015.

\begin{tabular}{ccccc}
\hline & LILACS & CINAHL & PUBMED & SCOPUS \\
\hline Produção encontrada & 02 & 09 & 89 & 292 \\
Não se encontra em português, inglês ou, espanhol & 00 & 00 & 00 & 11 \\
Não aborda a temática & 00 & 02 & 11 & 218 \\
Não possui intervenção & 00 & 02 & 64 & 32 \\
Não possui nível de evidência 1 ou 2 & 01 & 05 & 05 & 19 \\
Repetido & 00 & 00 & 01 & 00 \\
Total selecionado & 01 & 00 & 08 & 10 \\
\hline
\end{tabular}

A busca foi realizada por acesso online. Para a coleta dos dados, foi utilizado instrumento que abordou os seguintes itens: identificação do artigo, metodologia utilizada, local de realização da pesquisa, público-alvo das intervenções, conteúdo das intervenções e resultados encontrados.

Os artigos selecionados para este estudo foram analisados e os dados obtidos foram organizados e apresentados de acordo com as intervenções investigadas nos mesmos.

\section{RESULTADOS}

Inicialmente, foram encontrados 392 artigos, e após leitura dos resumos, foram excluídos 373, de acordo com os critérios pré-estabelecidos, sendo a amostra final constituída por 19 artigos.

Os artigos selecionados foram publicados em periódicos internacionais, específicos da área de drogas e álcool. Apenas um artigo foi publicado em periódico nacional, pertencente à área de enfermagem.

Os estudos foram desenvolvidos por pesquisadores afiliados a centros ou institutos de pesquisa nas áreas de álcool, drogas, saúde pública e psiquiatria, principalmente nos Estados Unidos (06), Suécia (03), Reino Unido (02), Austrália (02), Canadá (02), Alemanha (01), Japão (01), Nova Zelândia (01), Brasil (01), Holanda (01). Um artigo apresentou autores de dois países diferentes.
Os artigos selecionados foram distribuídos conforme a identificação do artigo, nível de evidência, público-alvo, intervenção pesquisada e principais resultados, como apresentado no Quadro 1. 
Quadro 1: Síntese dos artigos quanto a identificação do artigo, nível de evidência, público-alvo, intervenção pesquisada e principais resultados. Fortaleza, CE, Brasil, 2015.

\begin{tabular}{|c|c|c|c|c|}
\hline $\begin{array}{c}\text { Autor / Ano / } \\
\text { Periódico / Nível de } \\
\text { evidência }\end{array}$ & Título do artigo & Público & Intervenção & Principais resultados \\
\hline $\begin{array}{l}{ }^{(6)} \text { Cunningham et al / } \\
\text { PLoS One. / } 2012 \text { / } \\
\text { Nível II }\end{array}$ & $\begin{array}{c}\text { Ultra-Brief Intervention for Problem } \\
\text { Drinkers: Results from a Randomized } \\
\text { Controlled Trial }\end{array}$ & $\begin{array}{l}\text { Bebedores } \\
\text { problemáticos }\end{array}$ & $\begin{array}{l}\text { Intervenção ultra breve } \\
\text { (panfleto personalizado, } \\
\text { panfleto com informações } \\
\text { sobre álcool e ausência de } \\
\quad \text { panfleto) }\end{array}$ & $\begin{array}{l}\text { A intervenção breve modificada para um formato ultra breve pode ter um impacto } \\
\text { significativo para a saúde pública. }\end{array}$ \\
\hline $\begin{array}{l}{ }^{(7)} \text { Nehlin et al /. Addict } \\
\text { Sci Clin Pract. / } 2012 \text { / } \\
\text { Nível II }\end{array}$ & $\begin{array}{c}\text { Brief alcohol intervention in a psychiatric } \\
\text { outpatient setting: a randomized controlled } \\
\text { study }\end{array}$ & $\begin{array}{c}\text { Pacientes } \\
\text { ambulatoriais com } \\
\text { transtono mental }\end{array}$ & Intervenção Breve & $\begin{array}{l}\text { As IB podem promover redução de pontuação AUDIT em pequena escala em pacientes } \\
\text { com transtorno mental com o uso nocivo de álcool. }\end{array}$ \\
\hline $\begin{array}{l}{ }^{(8)} D^{\prime} \text { Onofrio et al. / } \\
\text { Ann Emerg Med. / } \\
2012 \text { / Nível II }\end{array}$ & $\begin{array}{l}\text { Brief Intervention Reduces Hazardous and } \\
\text { Harmful Drinking in Emergency Department } \\
\text { Patients }\end{array}$ & $\begin{array}{l}\text { Pacientes internos } \\
\text { em emergência }\end{array}$ & Intervenção Breve & $\begin{array}{l}\text { As IB podem reduzir o consumo de álcool em bebedores de risco e nocivo. Estes } \\
\text { resultados suportam o uso de intervenções breves em cenários de emergência. }\end{array}$ \\
\hline $\begin{array}{l}\text { (9) J Med Internet Res. } \\
\text { / } 2012 \text { / Nível II }\end{array}$ & $\begin{array}{l}\text { Comparison of Two Internet-Based } \\
\text { Interventions for Problem Drinkers: } \\
\text { Randomized Controlled Trial }\end{array}$ & $\begin{array}{l}\text { Bebedores } \\
\text { problema }\end{array}$ & $\begin{array}{l}\text { Intervenções baseadas em } \\
\text { internet }\end{array}$ & $\begin{array}{l}\text { A prestação do Centro de Ajuda ao Alcool forneceu maior benefício no curto prazo para } \\
\text { os bebedores-problema do que o observado no Check Your Drink (CYD). }\end{array}$ \\
\hline $\begin{array}{l}{ }^{(10)} \text { Helstrom et al. / } \\
\text { Addict Disord Their } \\
\text { Treat. / } 2014 \text { / Nível II }\end{array}$ & $\begin{array}{l}\text { Treating heavy drinking in primary care } \\
\text { practices: evaluation of a telephone-based } \\
\text { intervention program }\end{array}$ & $\begin{array}{l}\text { Usuários da } \\
\text { atenção primária }\end{array}$ & $\begin{array}{l}\text { Intervenção breve baseada no } \\
\text { telefone }\end{array}$ & $\begin{array}{l}\text { Houve redução na frequência do uso de álcool no grupo que recebeu a intervenção e } \\
\text { no grupo que recebeu o cuidado padrão. }\end{array}$ \\
\hline $\begin{array}{l}{ }^{(11)} \text { Vernon ML / J Subst } \\
\text { Abuse Treat. / 2010 / } \\
\text { Nível I }\end{array}$ & $\begin{array}{c}\text { A review of computer-based alcohol } \\
\text { problem services designed for the general } \\
\text { public. }\end{array}$ & Público em geral & $\begin{array}{l}\text { Programas baseado no } \\
\text { computador }\end{array}$ & Os individuos que completaram o programa parecem mostrar melhorias. \\
\hline $\begin{array}{l}{ }^{(12)} \text { Webb et al. / } \\
\text { Addiction. / } 2009 \text { / } \\
\text { Nível I }\end{array}$ & $\begin{array}{l}\text { A systematic review of work-place } \\
\text { interventions for alcohol-related problems }\end{array}$ & Trabalhadores & $\begin{array}{l}\text { Treinamento de habilidades } \\
\text { psicossociais, programas } \\
\text { baseados em internet, } \\
\text { materiais de autoajuda } \\
\end{array}$ & $\begin{array}{l}\text { Evidenciou-se que IB, as intervenções contidas em exames de saúde e estilo de vida, as } \\
\text { competências psicossociais de formação e encaminhamento de pares têm potencial } \\
\text { para produzir resultados benéficos. }\end{array}$ \\
\hline $\begin{array}{l}{ }^{(13)} \text { Freyer-Adam et al. / } \\
\text { Drug Alcohol Depend. } \\
\text { / } 2008 \text { / Nível II }\end{array}$ & $\begin{array}{l}\text { Brief alcohol intervention for general } \\
\text { hospital inpatients: a randomized controlled } \\
\text { trial }\end{array}$ & $\begin{array}{l}\text { Pacientes internos } \\
\text { em hospitais gerais }\end{array}$ & Intervenção Breve & $\begin{array}{l}\text { A intervenção não foi eficaz na redução do consumo de álcool ou no aumento do bem- } \\
\text { estar em } 12 \text { meses após a internação. Obteve-se um efeito positivo sobre prontidão } \\
\text { para mudar de beber e prontidão para buscar ajuda formal para problemas de } \\
\text { alcoolismo. }\end{array}$ \\
\hline $\begin{array}{l}{ }^{(14)} \text { Geshi et al. / Acta } \\
\text { Med. / } 2007 \text { / Nível II }\end{array}$ & $\begin{array}{l}\text { Effects of alcohol related health education } \\
\text { on alcohol and drinking behavior awareness } \\
\text { among Japanese junior college students: a } \\
\text { randomized controlled trial. } \\
\end{array}$ & $\begin{array}{l}\text { Estudantes } \\
\text { universitários }\end{array}$ & Educação em saúde & $\begin{array}{c}\text { A educação pode ser uma maneira efetiva para aumentar a consciência dos problemas } \\
\text { de saúde relacionados ao álcool, mas menos efetiva para mudança do comportamento } \\
\text { de beber entre estudantes japoneses. }\end{array}$ \\
\hline $\begin{array}{l}{ }^{(15)} \text { Ekman et al. / } \\
\text { Addictive Behav. / } \\
2011 \text { / Nível II }\end{array}$ & $\begin{array}{l}\text { Electronic screening and brief intervention } \\
\text { for risky drinking in Swedish university } \\
\text { students: a randomized controlled trial. }\end{array}$ & $\begin{array}{l}\text { Estudantes } \\
\text { universitários }\end{array}$ & $\begin{array}{l}\text { Feedback normativo e } \\
\text { intervenção breve }\end{array}$ & $\begin{array}{l}\text { Houve diminuição significativa do consumo médio semanal para o grupo intervenção. } \\
\text { Houve quedas significativas nos episódios de beber pesado ao longo do tempo em } \\
\text { ambos os grupos. A proporção de bebedores de risco diminuiu em cerca de um terço } \\
\text { no grupo controle e grupo intervenção. A intervenção breve genérica mais curta parece } \\
\text { ser tão eficaz quanto a mais longa, incluindo o feedback normativo. }\end{array}$ \\
\hline
\end{tabular}




\begin{tabular}{|c|c|c|c|c|}
\hline $\begin{array}{c}\text { Autor / Ano / } \\
\text { Periódico / Nível de } \\
\text { evidência }\end{array}$ & Título do artigo & Público & Intervenção & Principais resultados \\
\hline $\begin{array}{l}{ }^{(16)} \text { Cherpitel et al. / } \\
\text { Alcohol Clin Exp Res. / } \\
2010 \text { / Nível II }\end{array}$ & $\begin{array}{l}\text { Screening, brief intervention and referral to } \\
\text { treatment (SBIRT): } 12 \text { month outcomes of a } \\
\text { randomized controlled trial in a polish } \\
\text { emergency department }\end{array}$ & $\begin{array}{l}\text { Pacientes do setor } \\
\text { de emergência }\end{array}$ & $\begin{array}{l}\text { Triagem, intervenção breve e } \\
\text { referência ao tratamento }\end{array}$ & $\begin{array}{l}\text { Os participantes na condição de intervenção apresentaram melhora significativa em } \\
\text { todas as variáveis. }\end{array}$ \\
\hline $\begin{array}{l}{ }^{(17)} \text { Holloway et al. / } \\
\text { Addiction. / } 2007 \text { / } \\
\quad \text { Nível II }\end{array}$ & $\begin{array}{l}\text { The effect of brief interventions on alcohol } \\
\text { consumption among heavy drinkers in a } \\
\text { general hospital setting. }\end{array}$ & $\begin{array}{l}\text { Pacientes } \\
\text { internados em } \\
\text { hospitais gerais }\end{array}$ & $\begin{array}{l}\text { Autoeficácia, intervenção breve } \\
\text { e material de autoajuda }\end{array}$ & $\begin{array}{l}\text { O grupo de autoeficácia e o grupo que recebeu livreto de autoajuda tiveram maiores } \\
\text { reduções no consumo de álcool semanal. Não houve nenhuma evidência de que a } \\
\text { melhoria da autoeficácia foi superior ao livreto de autoajuda. As IB entregues no } \\
\text { hospital ajudam os bebedores pesados a reduzir o seu consumo de álcool }\end{array}$ \\
\hline $\begin{array}{l}{ }^{(18)} \text { Sinadinovic et al. / } \\
\text { Eur Addict Res. / } 2014 \\
\text { / Nível II }\end{array}$ & $\begin{array}{l}\text { Targeting individuals with problematic } \\
\text { alcohol use via web-based cognitive- } \\
\text { behavioral self-help modules, personalized } \\
\text { screening feedback or assessment only: A } \\
\text { randomized controlled trial. }\end{array}$ & $\begin{array}{l}\text { Usuários de } \\
\text { internet }\end{array}$ & $\begin{array}{l}\text { Programa de autoajuda versus } \\
\text { triagem e feedback, baseados } \\
\text { na internet }\end{array}$ & $\begin{array}{l}\text { A intervenção de autoajuda em combinação com outras intervenções foi mais eficaz na } \\
\text { mudança de uso de álcool do que apenas a triagem ou avaliação. }\end{array}$ \\
\hline $\begin{array}{l}{ }^{(19)} \text { Kypri et al / JAMA. } \\
2014 \text { / Nível II }\end{array}$ & $\begin{array}{l}\text { Web-based alcohol screening and brief } \\
\text { intervention for university students: A } \\
\text { randomized trial }\end{array}$ & $\begin{array}{l}\text { Estudantes } \\
\text { universitários }\end{array}$ & Triagem e intervenção breve & $\begin{array}{l}\text { A detecção do álcool baseada na web e programa de intervenção breve não produziu } \\
\text { reduções significativas na frequência e volume global de beber ou problemas } \\
\text { acadêmicos. }\end{array}$ \\
\hline $\begin{array}{l}{ }^{(20)} \text { Pereira et al. / } \\
\text { REBEN. / } 2013 \text { / Nível I }\end{array}$ & $\begin{array}{l}\text { Efetividade da intervenção breve para o uso } \\
\text { abusivo de álcool na atenção primária: } \\
\text { revisão sistemática }\end{array}$ & Não definido & Intervenção breve & $\begin{array}{l}\text { A intervenção breve é efetiva na redução da frequência e quantidade de álcool, mas } \\
\text { não foi possível definir se é mais eficaz em usuários com padrão nocivo ou } \\
\text { dependentes. }\end{array}$ \\
\hline $\begin{array}{l}\text { (21) Litten et al. / J } \\
\text { addict med. / } 2013 \text { / } \\
\text { Nível II }\end{array}$ & $\begin{array}{l}\text { A double-blind, placebo-controlled trial } \\
\text { assessing the efficacy of varenicicline } \\
\text { tartrate for alcohol dependence }\end{array}$ & $\begin{array}{c}\text { Usuários } \\
\text { dependentes de } \\
\text { álcool }\end{array}$ & Medicação & A medicação testada reduziu a fissura e o consumo de álcool. \\
\hline $\begin{array}{l}\text { (22) Gustafson et al. / } \\
\text { JAMA Psychiatry / } \\
2014 \text { / Nivel II }\end{array}$ & $\begin{array}{l}\text { A smartphone application to support } \\
\text { recovery from alcoholism: a randomized } \\
\text { controlled trial }\end{array}$ & $\begin{array}{c}\text { Usuários } \\
\text { dependentes de } \\
\text { álcool }\end{array}$ & Aplicativo para smartphone & $\begin{array}{l}\text { Os resultados sugerem que o uso do aplicativo pode apresentar benefícios para os } \\
\text { usuários com transtornos relacionados ao uso de álcool. }\end{array}$ \\
\hline $\begin{array}{l}{ }^{(23)} \text { Voogt et al. / } \\
\text { Alcohol Alcohol. / } \\
2013 \text { / Nível II }\end{array}$ & $\begin{array}{c}\text { The Effectiveness of the 'What Do You } \\
\text { Drink' Web-based Brief Alcohol Intervention } \\
\text { in Reducing Heavy Drinking among Students } \\
\text { A Two-arm Parallel Group Randomized } \\
\text { Controlled Trial }\end{array}$ & Estudantes & $\begin{array}{l}\text { Intervenção breve baseada na } \\
\text { internet }\end{array}$ & $\begin{array}{c}\text { A intervenção testada não foi eficaz em reduzir as medidas de álcool entre os } \\
\text { estudantes com consumo pesado. }\end{array}$ \\
\hline $\begin{array}{l}\text { (24) O’Donnell et al. / } \\
\text { Alcohol Alcohol. / } \\
2014 \text { / Nível I }\end{array}$ & $\begin{array}{l}\text { The Impact of Brief Alcohol Interventions in } \\
\text { Primary Healthcare: A Systematic Review of } \\
\text { Reviews }\end{array}$ & $\begin{array}{l}\text { Usuários da } \\
\text { atenção primária }\end{array}$ & Intervenção breve & $\begin{array}{l}\text { A intervenção breve apresenta efeitos positivos em diferentes ambientes culturais e } \\
\text { em grupos específicos da população. }\end{array}$ \\
\hline
\end{tabular}


Dos 19 artigos selecionados, as intervenções pesquisadas foram desenvolvidas em hospitais (06), em universidades (03), por meio da internet (03), por meio de telefone (02), ambulatório de psiquiatria (01), ambientes de trabalho (01), programas residenciais (01), atenção primária (01). Um artigo não especificou o local da intervenção.

\section{DISCUSSÃO}

$\mathrm{O}$ cuidado às pessoas que sofrem com o alcoolismo constitui desafio para gestores, profissionais de saúde, da educação e da assistência social. As ações de enfrentamento atualmente empregadas visam à promoção da saúde, prevenção ao consumo abusivo, ao cuidado integral e à criação de dispositivos que colaborem para a reabilitação de usuários de álcool e outras drogas.

Os achados desta revisão integrativa indicam que as intervenções mais utilizadas foram as intervenções breves (IB). As intervenções descritas nos estudos objetivaram a alteração de comportamento para modificar o padrão de consumo de álcool. A intervenção breve quando comparada aos cuidados usuais promove elevada redução no volume de álcool consumido por semana, na quantidade consumida e nos problemas com álcool ${ }^{(25)}$.

Tais achados são relevantes, porque as intervenções breves consistem em recursos simples, que poderão ser aplicadas por profissionais de qualquer área. As ações podem consistir em recomendações até recursos ofertados em um programa estruturado de tratamento ${ }^{(20)}$. Intervenções breves são entendidas como aquelas desenvolvidas por profissionais de saúde em um ambiente geral e envolvem uma interação individual ${ }^{(17)}$.

As intervenções breves descritas nos estudos foram empregadas em diversos ambientes. Observou-se tendência a desenvolver intervenções breves para pacientes dependentes ou não de álcool internos em hospitais. Estudos desenvolveram intervenções em setores de emergência, que constituem importantes locais para a implementação de triagem e intervenção precoce $^{(16,25)}$. As intervenções breves desenvolvidas em ambientes hospitalares são recursos simples, com baixo custo e que auxiliam usuários de álcool a reduzir o consumo $^{(17)}$.

A hospitalização isoladamente promove a redução do consumo de álcool, por isso as intervenções breves empregadas nesses ambientes estimulam maior redução nesse consumo quando comparadas ao tratamento usual $^{(13)}$. Dois estudos apontaram que a intervenção breve foi considerada intervenção eficaz para homens usuários de álcool, pois reduziu o consumo diário ou semanal ${ }^{(16,26)}$. As IB, também, foram empregadas em mulheres com diagnóstico de depressão pós-parto observando-se seu efeito positivo na sintomatologia depressiva durante esse período ${ }^{(27)}$.

Esses resultados demonstram que a intervenção breve pode ser aplicada para ambos os gêneros, o que a torna uma relevante estratégia, pois estudo identificou que há diferenças relacionadas ao gênero no que diz respeito ao abuso/uso nocivo e dependência ao álcool, como também, se deve considerar as diferenças entre os gêneros no tratamento para problemas relacionados ao álcool $^{(28)}$.

Ademais, o uso da intervenção breve associado a triagem eletrônica promoveram redução significativa na média de consumo semanal de álcool, como também reduções significativas no consumo pesado esporádico do álcool $^{(15)}$. Essas intervenções apresentam impacto positivo na taxa de mortalidade relacionada ao abuso de álcool $^{(20)}$. Há estimativa de que em 2012, aproximadamente 3,3 milhões de mortes foram causadas pelo consumo de álcool, o que corresponde a 5,9\% de todas as mortes no mundo ${ }^{(1)}$. As principais causas de mortes relacionadas ao consumo de álcool são a violência e acidentes.

Evidenciaram-se, também, intervenções para o público jovem, em que se empregou a intervenção breve e triagem para essa clientela. Acredita-se que a motivação para trabalhar com essa população esteja fundamentada 
no fato de que o público jovem se encontra mais suscetível e vulnerável ao uso de substâncias psicoativas $^{(29)}$. Durante a adolescência costumam ocorrer os primeiros episódios de abuso de bebidas alcoólicas ou outras drogas. As bebidas alcoólicas e o tabaco foram citados como as substâncias mais consumidas pelos adolescentes $^{(30)}$.

Alguns estudos revisados apresentaram as intervenções pautadas nos recursos da internet, as quais foram avaliadas como relevantes para a redução da média semanal do consumo do álcool em adultos jovens, com redução no status de abuso de álcool para o status consumo de não risco. Houve redução significante no ato de beber pesado, ou seja, no consumo de várias doses ao beber $^{(15,31)}$. As orientações sobre o padrão de consumo podem variar consideravelmente, mas o uso de risco pode, muitas vezes, ser definido como um alto consumo semanal ou um alto consumo em uma única ocasião (binge drinking) ${ }^{(32)}$.

Os estudos apresentaram sites que continham informações sobre o álcool e a triagem eletrônica, em que o usuário poderia definir seu padrão de consumo de álcool e obter informações sobre seu estado de saúde ${ }^{(15,31)}$. Nesta perspectiva, o uso da informação se torna uma intervenção que pode auxiliar o usuário em sua tomada de decisão.

Um estudo identificou que o impacto da intervenção de experiências personalizadas baseadas na web sobre o consumo de álcool foi significativo. Esta estratégia é importante para conduzir indivíduos com problemas com o álcool ao tratamento, os quais, geralmente, apresentam resistência para procurar ajuda para lidar com a problemática que os envolve ${ }^{(31)}$. Ademais, há o preconceito e estigma em relação ao alcoolista que afetam seu tratamento e reabilitação( ${ }^{(33)}$

Os instrumentos de triagem baseados na internet podem ser úteis aos profissionais, uma vez que os resultados obtidos nos testes podem ser enviados online para os serviços de referência em saúde. Além disso, com a expansão do uso pela população em geral, a internet permite alcançar maior número de pessoas e constitui a maior fonte de informações às pessoas que a acesse em qualquer parte do mundo ${ }^{(34)}$. Ademais, o acesso a internet é comum entre pessoas com problemas relacionados ao uso de álcool e outras drogas ${ }^{(35)}$.

Um dos estudos revisados foi realizado com pacientes de hospital psiquiátrico, em que foi utilizado o aconselhamento, a intervenção individual e o tratamento em grupo. Neste, os pacientes mostraram maior taxa de participação( ${ }^{(10)}$. Nos grupos de autoajuda, os participantes buscam resolver seus problemas relacionados ao álcool. A participação nesses grupos contribui para o bem-estar físico, mental e social, tendo em vista o acolhimento e a partilha de experiências vivenciadas nos grupos ${ }^{(36)}$.

Comparando-se grupos que receberam o cuidado usual, material de autoajuda e aprimoramento da autoeficácia, observou-se que os dois últimos grupos obtiveram reduções no consumo semanal de álcool ${ }^{(17)}$. Este resultado propõe que para enfrentar a problemática do álcool, a diversidade de materiais, como panfletos de autoajuda, pode ser eficaz, associada às intervenções breves e aconselhamento.

Um dos estudos revisados teve como foco a educação em saúde para estudantes. No programa foi utilizado vídeo e palestra de ex-usuário de álcool, destacando a luta contra a dependência e o caminho para recuperação do alcoolismo. Essa estratégia é importante nos programas de educação em saúde, os quais têm contribuído para o aumento do conhecimento de estudantes sobre problemas relacionados ao álcool ${ }^{(13)}$.

Vale ressaltar que o alcoolismo consiste em um transtorno crônico e que sua ocorrência é resultado de longo período de consumo pesado de bebida alcoólica e que os estudantes, geralmente, apresentam, neste momento da vida, uso ocasional de bebidas, não sendo, portanto, considerados alcoolistas, mas podem ser considerados consumidores de risco. Estudo ressalta a necessidade da realização de ações voltadas para a prevenção do consumo de álcool entre estudantes, 
objetivando a diminuição da prevalência de sua utilização( ${ }^{(37)}$.

As ações de educação em saúde são relevantes para auxiliar no incremento da qualidade de vida de usuários dos serviços. O profissional de saúde, especificamente o enfermeiro deve desenvolver ações de prevenção de agravos decorrentes do consumo de álcool, por meio de intervenções educativas e informativas em escolas, ambientes de trabalho, serviços de atenção à saúde ${ }^{(38)}$. 0 desafio se encontra em desenvolver atividades que estimulem a conscientização pela valorização da vida. 0 enfermeiro ao desenvolver tais atividades deve estimular a tomada de consciência dos sujeitos que estão envolvidos no processo educativo ${ }^{(39)}$. A educação em saúde envolve a prevenção da doença, como também a promoção da saúde. Nesta perspectiva, o alcoolismo deve ser abordado quanto a prevenção e promoção da saúde ${ }^{(33)}$.

Dessa forma, sugere-se que sejam destacados em programas de educação em saúde a promoção da saúde, as consequências do consumo excessivo de álcool e a intoxicação aguda do álcool $^{(14)}$. A promoção da saúde e prevenção do abuso do álcool podem, também, ser realizadas em ambientes de trabalho. Corrobora estudo que destacou intervenções realizadas em ambientes de trabalho para redução do abuso de álcool, por meio do uso de materiais de autoajuda, dos programas baseados na internet, treinamento de habilidades psicossociais ${ }^{(12)}$.

Os parâmetros utilizados nos estudos para mensurar o consumo de álcool após as intervenções devem ser ressaltados. Os estudos utilizaram diferentes medidas, como doses padrão consumidas ao dia ou semanal; AUDIT (Alcohol Use Disorders Identification Test) para avaliar problemas de consumo; relato de beber pesado; dentre outros.

O AUDIT se mostrou um instrumento adequado, por facilitar o processo de triagem com utilização a nível mundial, de fácil aplicação e ligado a intervenções breves. Por meio desta ferramenta de triagem podem ser identificadas quatro zonas de risco relacionadas ao consumo de álcool, a saber: uso de risco baixo ou zona de risco I (0 a 7 pontos), uso nocivo ou zona de risco II (8 a 15 pontos), uso nocivo ou zona de risco III (16 a 19 pontos) e provável dependência ou zona de risco IV (20 a 40 pontos) ${ }^{(40)}$.

Ressalta-se a importância em se elaborar e utilizar instrumentos específicos para a avaliação do consumo de álcool, como também a sua validação para diferentes grupos populacionais. Dessa forma, observa-se que são necessários estudos que forneçam conhecimentos para essas lacunas existentes.

As intervenções para redução do alcoolismo são diversificadas e buscam atender às necessidades da clientela assistida, como também ao local, no qual se pretende desenvolvê-las. As intervenções podem ser desenvolvidas por profissionais de saúde, especificamente enfermeiros, pois apresentaram evidências de sua eficácia no enfrentamento ao alcoolismo.

\section{CONSIDERAÇÕES FINAIS}

A revisão integrativa revelou que estudos estão sendo desenvolvidos com objetivo de avaliar intervenções eficazes para a redução do abuso de álcool. Os tipos de intervenção, locais de aplicação, público-alvo variaram consideravelmente, refletindo as múltiplas estratégias que podem ser utilizadas para enfrentar tal problemática. Destas, destacaram-se as intervenções breves que se mostraram simples e com baixo custo, o que é relevante para os serviços de saúde que funcionam com orçamentos limitados. Portanto, as intervenções breves constituem as melhores intervenções para redução do abuso de álcool, no entanto nenhuma intervenção isoladamente será capaz de reduzir significativamente os padrões de consumo do álcool por um longo período de tempo.

Nenhum estudo apontou a cessação do uso da bebida alcoólica com as intervenções avaliadas. No entanto, os resultados demonstraram redução no consumo de álcool significativamente maior do que os cuidados usuais e por 
maior período de tempo. Ademais, revelaram aumento na disponibilidade para mudança do ato de beber. Esses resultados são importantes, tendo em vista os desafios implicados no tratamento e na reabilitação de alcoolistas.

No contexto em que as políticas públicas tornam-se mais presentes para enfrentar a problemática do alcoolismo, é imperativo buscar por melhores evidências científicas para lidar com esta situação.

\section{REFERÊNCIAS}

1. World Health Organization. Global status report on alcohol and health - 2014. Geneva, Switzerland; 2014 [Acesso em 17 mar 2015]. Disponível em:

http://www.cisa.org.br/artigo/4429/relatorio-global-sobrealcool-saude-2014.php..

2. Centro Brasileiro de Informação sobre Drogas Psicotrópicas. Universidade Federal de São Paulo. II Levantamento domiciliar sobre o uso de drogas psicotrópicas no Brasil: estudo envolvendo as 108 maiores cidades do país: 2005. São Paulo: Páginas \& Letras; 2006.

3.II Levantamento nacional de álcool e drogas. Resultados preliminares. 2013. Disponível em:

http://inpad.org.br/LENAD/. Acesso em 17 mar. 2015.

4. Mendes KDS, Silveira RCCP, Galvão CM. Revisão integrativa: método de pesquisa para a incorporação de evidências na saúde e na enfermagem. Texto Contexto Enferm. 2008;17:75864.

5.Polit DF, Beck CT. Fundamentos de pesquisa em enfermagem: avaliação de evidências para a prática da enfermagem. 7 ed. Porto Alegre: Artmed; 2011.

6. Cunningham JA, Neighbors C, Wild C, Humphreys K. UltraBrief Intervention for Problem Drinkers: Results from a Randomized Controlled Trial. PLoS One [Internet]. 2012 [acesso em: 20 dez 2013];7(10):e48003. Disponível em:

http://dx.doi.org/10.1371/journal.pone.0048003.

7. Nehlin C, Grönbladh L, Fredriksson A, Jansson L. Brief alcohol intervention in a psychiatric outpatient setting: a randomized controlled study. Addict Sci Clin Pract. [Internet]. 2012 [acesso em: 20 dez 2013];7:23. Disponível em:

http://www.ascpjournal.org/content/7/1/23

8. D'Onofrio G, Fiellin DA, Pantalon MV, Chawarski MC, Owens $\mathrm{PH}$, Degutis LC, et all. Brief Intervention Reduces Hazardous and Harmful Drinking in Emergency Department Patients. Ann Emerg Med.[Internet] 2012 [Acesso em 20 dez 2013];60(2). Disponivel em:

http://dx.doi.org/10.1016/j.annemergmed.2012.02.006

9. Comparison of Two Internet-Based Interventions for Problem Drinkers: Randomized Controlled Trial. J Med Internet Res. [Internet]. 2012 [acesso em: 20 dez 2013];14(4):e107. Disponível em: http://dx.doi.org/10.2196/jmir.2090.
Como limitação deste estudo destaca-se, a heterogeneidade entre os estudos, quanto ao públicoalvo e intervenções investigadas, o que impossibilitou a análise comparativa entre seus resultados.

A revisão é relevante para a prática clínica, pois colabora com a discussão acerca das intervenções eficazes para redução do consumo de álcool, além de apresentar opções de estratégias para os profissionais de saúde, que lidam com a problemática.

10. Helstrom AW, Ingram E, Wang W, Small D, Klaus J, Oslin D. Treating heavy drinking in primary care practices: evaluation of a telephone-based intervention program. Addict Disord Their Treat [Internet], 2014 [acesso em: 16 mar. 2015]; 13(3):101109.

11. Vernon ML. A review of computer-based alcohol problem services designed for the general public. J Subst Abuse Treat. [Internet]. 2010 [acesso em: $20 \mathrm{dez}$ 2013];38:203-11. Disponível em: http://dx.doi.org/10.1016/j.jsat.2009.11.001 12. Webb G, Shakeshaft A, Sanso-Fischer R, Havard A. A systematic review of work-place interventions for alcoholrelated problems. Addiction. [Internet]. 2009 [acesso em: 20 dez 2013];104:365-77. Disponível em:

http://dx.doi.org/10.1111/j.1360-0443.2008.02472.x. 13. Freyer-Adam J, Coder B, Baumeister SE, Bischof G, Riedel J, Paatsch $\mathrm{K}$ et al. Brief alcohol intervention for general hospital inpatients: a randomized controlled trial. Drug Alcohol Depend. [Internet]. 2008 [acesso em: $20 \mathrm{dez}$ 2013];93:233-43. Disponível em:

http://dx.doi.org/10.1016/j.drugalcdep.2007.09.016. 14. Geshi M, Hirokawa K, Taniguchib T, Fujib Y, Kawakamic N. Effects of alcohol related health education on alcohol and drinking behavior awareness among Japanese junior college students: a randomized controlled trial. Acta Med. [Internet]. 2007 [acesso em: 20 dez 2013];61:345-54. Disponível em: http://www.ncbi.nlm.nih.gov/pubmed/18183080 15. Ekman DS, Andersson A, Nielsen P, Stahlbrandt H, Johansson AL, Bendtsen P. Electronic screening and brief intervention for risky drinking in Swedish university students: a randomized controlled trial. Addictive Behav. [Internet]. 2011 [acesso em: 20 dez 2013];36:654-9. Disponível em: http://dx.doi.org/10.1016/j.addbeh.2011.01.015 16. Cherpitel CJ, Korcha RA, Moskalewicz J, Swiatkiewicz G, Ye $Y$, Bond J. Screening, brief intervention and referral to treatment (SBIRT): 12 month outcomes of a randomized controlled trial in a polish emergency department. Alcohol Clin Exp Res. [Internet]. 2010 [acesso em: 20 dez 2013];34:1922-28. Disponível em: http://dx.doi.org/10.1111/j.15300277.2010.01281.x.

17. Holloway AS, Watson HE, Arthur AJ, Starr G, McFadyen AK, McIntosh J. The effect of brief interventions on alcohol 
consumption among heavy drinkers in a general hospital setting. Addiction. [Internet]. 2007 [acesso em: $20 \mathrm{dez}$ 2013];102:1762-70. Disponível em: http://dx.doi.org/10.1111/j.1360-0443.2007.01968.x 18. Sinadinovic K, Wennberg $P$, Johansson M, Berman AH. Targeting individuals with problematic alcohol use via webbased cognitive-behavioral self-help modules, personalized screening feedback or assessment only: A randomized controlled trial. Eur Addict Res. [Internet]. 2014 [acesso em: 16 mar 2015];20(6): 305-18. Disponível em:

http://dx.doi.org/10.1159/000362406.

19. Kypri K, Vater T, Bowe SJ, Saunders JB, Cunningham JA, Horton NJ, et al. Web-based alcohol screening and brief intervention for university students: A randomized trial. JAMA. [Internet]. 2014 [acesso em: 16 mar 2015];311(12): 1218-24. Disponível em:

http://jama.jamanetwork.com/article.aspx?articleid=1849990. 20. Pereira MO, Anginoni BM, Ferreira NC, Oliveira MAF, Vargas D, Colvero LA. Efetividade da intervenção breve para o uso abusivo de álcool na atenção primária: revisão sistemática. Rev Bras Enferm. [Internet]. 2013 [acesso em: $17 \mathrm{mar}$ 2015];66(3): 420-8. Disponível em:

http://www.scielo.br/pdf/reben/v66n3/a18v66n3.pdf. 21. Litten RZ, Ryan ML, Fertig JB, Falk DE, Johnson B, Dunn KE et al. A double-blind, placebo-controlled trial assessing the efficacy of varenicicline tartrate for alcohol dependence. J addict med. [Internet]. 2013 [acesso em: 17 mar 2015];7(4):277-286. Disponível em: http://dx.doi.org/10.1097/ADM.0b013e31829623f4. 22. Gustafson DH, McTavish FM, Chih MY, Atwood AK, Johnson RA, Boyle MG. A smartphone application to support recovery from alcoholism: a randomized controlled trial. JAMA Psychiatry. [Internet]. 2014 [acesso em: 17 mar. 2015];71(5): 566-572. Disponível em: doi:10.1001/jamapsychiatry.2013.4642.

23. Voogt CV, Poelen EAP, Kleinjan M, Lemmers LACJ, Engels RCME. The Effectiveness of the 'What Do You Drink' Webbased Brief Alcohol Intervention in Reducing Heavy Drinking among Students A Two-arm Parallel Group Randomized Controlled Trial. Alcohol Alcohol. [Internet] 2013 [acesso em: 17 mar. 2015]; 48(3):312-21. Disponível em: doi:10.1093/alcalc/ags133.

24. O'Donnell A, Anderson P, Newbury-Birch D, Schulte B, Schmidt $C$, Reimer J, et al. The Impact of Brief Alcohol Interventions in Primary Healthcare: A Systematic Review of Reviews. Alcohol Alcohol. [Internet]. 2014 [acesso em: 17 mar. 2015];49(1): 66-78. Disponível em: doi: 10.1093/alcalc/agt170. 25. Field CA, Caetano R. The effectiveness of brief intervention among injuried patients with alcohol dependence: who benefits from brief interventions? Drug Alcohol Depend. [Internet]. 2010 [acesso em: $20 \mathrm{dez} 2013$ ];111(1-2):13-20. Disponível em: http://dx.doi.org/

10.1016/j.drugalcdep.2009.11.025

26. Noknoy S, Rangsin R, Saengcharnchai P, Tantibhaeshygkul $U$, McCambridges J. RCT of effectiveness of motivacional enhancement therapy delivered by nurses for hazardous drinkers in primary care units in Thailand. Alcohol Alcohol.
[Internet]. 2010 [acesso em: 20 dez. 2013];45:263-70. Disponível em: http://dx.doi.org/10.1093/alcalc/agq013. 27. Wilton G, Moberg DP, Fleming MF. The effect of Brief Alcohol Intervention on Postpartum Depression. MCN Am J Matern Child Nurs. [Internet]. 2009 [acesso em: $20 \mathrm{dez}$ 2013];34(5):297-302. Disponível em: http://dx.doi.org/10.1097/01.NMC.0000360422.06486.c4. 28. Berenzon S, Robles R, Reed GM, Medina-Mora ME. Questões relacionadas ao gênero no diagnóstico e classificação de transtornos por uso de álcool entre pacientes mexicanos que buscam serviços especializados. Rev. Bras. Psiquiatr. [Internet]. 2011 [acesso 18 abr 2015];33(Suppl 1):s109-s116. Disponível em: http://dx.doi.org/10.1590/S1516-

44462011000500008

29. Martins RA, Manzatto AJ, Cruz LN, Poiate SMG, Scarin ACCF. Utilização do Alcohol Use Disorders Identification Test (Audit) para Identificação do Consumo de Álcool entre Estudantes do Ensino Médio. Interam. J. Psychol. 2008;42(2):307-16.

30. Centro Brasileiro de Informações sobre drogas psicotrópicas. Universidade Federal de São Paulo. VI Levantamento Nacional sobre o Consumo de Drogas Psicotrópicas entre Estudantes do Ensino Fundamental e Médio das Redes Pública e Privada de Ensino nas 27 Capitais Brasileiras. Brasília: SENAD - Secretaria Nacional de Políticas sobre Drogas; 2010.

31. Cunningham JA, Wild TC, Cordingley J, Mierlo TV, Humphreyes $\mathrm{K}$. Twelve-month follow up results from a randomized controlled trial of a brief personalized feedback intervention for problems drinkers. Alcohol Alcohol. [Internet]. 2010 [acesso em: 10 jan 2014];45:258-62. Disponível em: http://dx.doi.org/10.1093/alcalc/agq009.

32. Gajecki M, Berman AH, Sinadinovic K, Rosendahl I, Andersson C. Mobile phone brief intervention applications for risky alcohol use among university students: a randomized controlled study. Addict Sci Clin Pract. [Internet]. 2014 [acesso 24 abr 2015]; 9(1): 2-12. Disponível em:

http://www.ncbi.nlm.nih.gov/pmc/articles/PMC4091647/?tool =pubmed.

33. Silva SED, Padilha MICS, Borenstein MS, Spricigo JS. Alcoolismo e a produção científica da enfermagem brasileira: uma análise de 10 anos. Rev. Eletr. Enf. [Internet].

2011;13(2):276-84. Disponível em: http://dx.doi.org/10.5216/ree.v13i2.9616.

34. Miranda LM, Farias SF. Contributions from the internet for elderly people: a review of the literature. Interface Comunic. Saúde Educ. [Internet]. 2009 [acesso em: $22 \mathrm{dez}$ 2013];13(29):383-94. Disponível em:

http://www.scielo.br/scielo.php?pid=S141432832009000200011\&script=sci_arttext

35. Kay-Lambkin F, White A, Baker AL, Kavanagh DJ, Proudfoot J, Drennan J, et al. Assessment of function and clinical utility of alcohol and other drug web sites: An observational, qualitative study. BMC Public Health. [Internet]. 2011 [acesso em: $21 \mathrm{dez}$ 2013];11:277. Disponível em: http://www.biomedcentral.com/1471-2458/11/277. 
36. Lima HP, Braga VAB. Grupo de autoajuda como modalidade de tratamento para pessoas com dependência de álcool. Texto contexto - enferm. [Internet]. 2012 [acesso em: $18 \mathrm{abr}$ 2015];21(4): 887-895. Disponível em:

http://www.scielo.br/scielo.php?script=sci_arttext \&pid=S0104 $-07072012000400020 \&$ Ing $=$ en.

http://dx.doi.org/10.1590/S0104-07072012000400020.

37. Campos JADB, Almeida JC, Garcia PPNS, Faria JB. Consumo de álcool entre estudantes do ensino médio do município de Passos - MG. Ciênc. saúde coletiva [Internet]. 2011 [acesso em: 21 dez 2013];16(12):4745-54. Disponível em:

http://www.scielo.br/scielo.php?script=sci_arttext\&pid=S1413 $-81232011001300023 \& \operatorname{lng}=p t \& n r m=i s o \& t \operatorname{lng}=p t$

38. Jomar RT, Abreu AMM. Produção científica sobre consumo de bebidas alcoólicas em periódicos brasileiros de Enfermagem. Rev Enferm UERJ. 2011;9(3):491-6.

39. Luna IT, Silva KL da, Dias FLA, Freitas MMC, Vieira NFC, Pinheiro PNC. Ações educativas desenvolvidas por enfermeiros brasileiros com adolescentes vulneráveis às DST/AIDS. Cienc. enferm. [Internet]. 2012 [acesso em: $21 \mathrm{dez}$ 2013];18(1):43-55. Disponível em:

http://www.scielo.cl/scielo.php?script=sci_arttext\&pid=S071795532012000100005\&lng=pt. doi: 10.4067/S0717-

\section{5 .}

40. Babor TF, Higgins-Biddle JC, Saunders JB, Monteiro MG. The Alcohol Use Disorders Identification Test: Guideline for use in Primary Care. 2nd Edition. Geneva: World Health Organization (WHO); 2001.

Recebido: 02/04/2014.

Aceito: $11 / 03 / 2015$.

Publicado: 30/09/2015. 